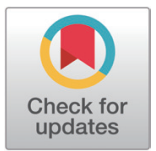

Received: Dec 2, 2020

Revised: Dec 29, 2020

Accepted: Dec 29, 2020

*Corresponding author

Min Kyu Kim

Division of Animal and Dairy Science,

College of Agriculture and Life Science,

Chungnam National University,

Daejeon 34134, Korea.

Tel: +82-42-821-5773

E-mail: kminkyu@cnu.ac.kr

Copyright (? 2021 Korean Society of Animal Sciences and Technology.

This is an Open Access article distributed under the terms of the Creative Commons Attribution

Non-Commercial License (http:// creativecommons.org/licenses/by$\mathrm{nc} / 4.0 /$ ) which permits unrestricted non-commercial use, distribution, and reproduction in any medium, provided the original work is properly cited.

ORCID

Ji Hye Lee

https://orcid.org/0000-0003-3645-5632

Miyoun Yoo

https://orcid.org/0000-0001-5516-1512

Sang Mee Lee

https://orcid.org/0000-0001-9425-8077

Soon Jae Park

https://orcid.org/0000-0003-4584-4891

Tae Young Kil

https://orcid.org/0000-0003-4143-449X

Min Kyu Kim

https://orcid.org/0000-0002-9259-8219

Competing interests

No potential conflict of interest relevant to this article was reported.

\section{Toxicity of the recombinant human hyaluronidase ALT-BC4 on embryonic development}

\author{
Ji Hye Lee ${ }^{1}$, Miyoun Yoo ${ }^{2}$, Sang Mee Lee ${ }^{2}$, Soon-Jae Park ${ }^{2}$, Tae Young Kil ${ }^{3}$ \\ and Min Kyu Kim ${ }^{1,4 *}$ \\ ${ }^{1}$ Division of Animal and Dairy Science, College of Agriculture and Life Science, Chungnam National \\ University, Daejeon 34134, Korea \\ ${ }^{2}$ Alteogen, Daejeon 34054, Korea \\ ${ }^{3}$ Department of Social Welfare, Joongbu University, Geumsan 32713, Korea \\ ${ }^{4}$ MK Biotech, Daejeon 34134, Korea
}

\section{Abstract}

Cumulus-oocyte complexes (COCs), which contain immature oocytes, are matured in vitro for in vitro embryo production. Oocyte and cumulus cells are then separated using hyaluronidase. To date, there have only been a few reported cases of the toxic effects of hyaluronidase on porcine oocytes. The aim of this study was to compare the effects of bovine testis-derived hyaluronidase and recombinant human hyaluronidase on oocyte denudation and quality. Porcine COCs were matured for $44 \mathrm{~h}$ and denuded using different hyaluronidase concentrations and exposure times. Then, oocytes were activated by electrical parthenogenesis. In experiment 1, COCs were denuded using bovine-derived, ovine-derived (Hirax), and human recombinant (ALT-BC4) hyaluronidases for 10 and $20 \mathrm{~min}$. In experiment 2, bovine-derived and human recombinant (ALT-BC4 and ICSI Cumulase ${ }^{\circledR}$ ) hyaluronidases were used to denude the COCs for 2 and $20 \mathrm{~min}$. In both experiments the oocytes were all completely denuded, and there was no degeneration. Rate of embryo development was significantly increased in group treated ALT-BC4 for 2 min and not significantly different in other treatment groups. In general it slightly decreased with longer exposure times. These results have confirmed that different sources of hyaluronidase do not have detrimental effects on the quality of porcine oocytes and suggest that the human recombinant hyaluronidase ALT-BC4 is suitable for oocyte denudation with an increased blastocyst rate.

Keywords: Hyaluronidase, Porcine, Cumulus-oocyte complex (COC), Parthenogenesis, Embryonic development

\section{INTRODUCTION}

Hyaluronidase is an enzyme that catalyzes the degradation of hyaluronan and hyaluronic acid (HA) The hydrolysis of HA by hyaluronidase is important for the switch from the morphogenetic and proliferative stages of embryonic development to the differentiation stages [1]. After sperm reach the cumulus-oocyte complex (COC), hyaluronidase is released by the acrosome of the sperm and this aids in fertilization, as it digests the hyaluronan in the extracellular matrix of the cumulus cells [2] which 
Funding sources

This work was funded by Alteogen, Korea.

Acknowledgements

We would like to thank Editage (www.

editage.co.kr) for English language editing.

Availability of data and material Upon reasonable request, the datasets of this study can be available from the corresponding author.

Authors' contributions

Conceptualization: Lee JH, Yoo MY

Data curation: Lee JH, Kim MK.

Formal analysis: Lee JH, Kim MK.

Methodology: Lee JH, Yoo MY.

Software: Lee JH, Kil TY.

Validation: Yoo MY, Lee SM, Park SJ.

Investigation: Lee JH, Kim MK.

Writing - original draft: Lee $\mathrm{JH}$.

Writing - review \& editing: Lee JH, Kim MK.

Ethics approval and consent to participate This article does not require IRB/IACUC approval because there are no human and animal participants. allows for the sperm to bind to the oocyte. In humans, hyaluronidase is considered to be necessary for the success of in vitro fertilization, as human semen hyaluronidase activity was found to be significantly related to the fertilization rates of oocytes in vitro [3]. Hyaluronidase is consequently used for oocyte denudation of the COC before intracytoplasmic sperm injection (ICSI), somatic cell nuclear transfer (SCNT), and microinjection. The removal of the cumulus cells from the oocytes is thus a necessary process for the identification of matured oocytes and experimental ease [4].

Oocytes are traditionally denuded using bovine testis-derived hyaluronidase that affects the quality of oocytes and embryos [5]. In hospitals, human recombinant hyaluronidase was commercially used as an alternative to bovine-derived hyaluronidase owing to its purity, safety, and effectiveness. It enhances drug delivery in addition to removing the cumulus cells [6], but decreases the efficiency of cumulus dispersal when compared with the bovine-derived hyaluronidase. Animal embryology labs thus prefer commercial bovine hyaluronidase [7].

In mice, oocytes that were denuded using hyaluronidase had reduced fertilization when compared with the COCs, due to the irreversible modification of the sperm receptors in the zona pellucida [8]. Ishizuka et al. have shown that prolonged treatment with hyaluronidase decreases the quality of mouse oocytes and that a shorter exposure time increases fertilization and developmental ability [9]. Furthermore, human oocytes treated with hyaluronidase have also been shown to have decreased oocyte survival, fertilization rates, and developmental ability following ICSI [4,5,10]. In pigs, the effect of different denuding procedures on oocyte damage and embryo development has previously been investigated [11]. However, the effects of hyaluronidase on porcine oocytes are not currently known.

ALT-BC4 was developed by Alteogen (Daejeon, Korea) and is based on Alteogen's Hybrozyme ${ }^{\circledR}$ technology. Hybrozyme technology utilizes domain swapping between two structurally similar enzymes using homology modeling, and maintains the inherent catalytic mechanisms but improves the thermal stability of the target enzyme. ALT-BC4 is a recombinant human hyaluronidase based on $\mathrm{PH} 20$, but the structural domain of $\mathrm{PH} 20$ is replaced with the corresponding human Hyal 1 domain and additional amino acids are deleted in the $\mathrm{C}$ - and $\mathrm{N}$-termini. It is generally expressed as a glycoprotein in Chinese hamster ovary $(\mathrm{CHO})$ cells $(\mathrm{CHO}$ DG44 - dihydrofolate reductase deficient [DHFR-]). According to the results of previous research, ALT-BC4 has improved thermal and $\mathrm{pH}$ stability when compared to recombinant human hyaluronidase $\mathrm{PH} 20$ enzyme (rHuPH20). The effect of ALT-BC4 on the oocytes has not been studied.

In this study, we have investigated the toxic effects of hyaluronidase on porcine embryonic development. COCs were treated with bovine testis-derived and recombinant human hyaluronidases for mechanical denudation via pipetting. The oocytes were subjected to parthenogenetic activation, and then oocyte denudation and developmental ability were analyzed.

\section{MATERIALS AND METHODS}

All chemicals were purchased from Sigma-Aldrich (St. Louis, MO, USA), unless otherwise stated. ALT-BC4 was supplied by Alteogen.

\section{Oocyte collection and in vitro maturation}

Porcine ovaries were collected from a local slaughterhouse and transported within 2-3 h to the laboratory in physiological saline (0.9\%) supplemented with $1 \%$ penicillin/streptomycin at $25^{\circ} \mathrm{C}$. The ovaries were washed in saline and then antral follicles $3-8 \mathrm{~mm}$ in diameter were aspirated using a $10 \mathrm{~mL}$ syringe with an 18-gauge needle. The follicular fluid was precipitated for $20 \mathrm{~min}$ and 
the sediments were washed twice with saline supplemented with 1\% penicillin/streptomycin and $0.5 \%$ fetal bovine serum (Gibco, Grand Island, NE, USA). COCs with homogenous cytoplasm and at least three layers of cumulus cells were selected. The COCs were washed with in vitro maturation (IVM) medium. The IVM medium was medium 199 (Gibco) supplemented with $2.5 \mathrm{mM}$ fructose, $0.6 \mathrm{mM}$ L-cysteine, $0.9 \mathrm{mM}$ sodium pyruvate, $75 \mu \mathrm{g} / \mathrm{mL}$ kanamycin, $10 \%$ (v/ v) porcine follicular fluid $(\mathrm{PFF}), 10 \mathrm{ng} / \mathrm{mL}$ epidermal growth factor $(\mathrm{EGF}), 10 \mathrm{IU} / \mathrm{mL}$ pregnant mare serum gonadotropin (PMSG, Merck Animal Health, Madison, NJ, USA), and $10 \mathrm{IU} / \mathrm{mL}$ human chorionic gonadotropin (hCG, Merck Animal Health). There were 50 COCs per well (4well multi-dishes, Thermo Scientific, Rochester, NY, USA) and they were cultured in $500 \mu \mathrm{L}$ IVM medium for $22 \mathrm{~h}$ at $38.5^{\circ} \mathrm{C}$ with $5 \% \mathrm{CO}_{2}$. Then, COCs were cultured for an additional $22 \mathrm{~h}$ in IVM medium without hormones.

\section{Parthenogenetic activation and in vitro culture}

After IVM for $44 \mathrm{~h}$, the COCs were denuded by pipetting with hyaluronidase according to the experimental design. Among the denuded oocytes those that had formed the first polar body were selected. The mature oocytes were then washed 3 times in electrical activation medium containing $0.28 \mathrm{M}$ mannitol, $0.5 \mathrm{mM}$ HEPES, $0.1 \mathrm{mM} \mathrm{MgSO}$, $0.1 \mathrm{mM} \mathrm{CaCl}_{2}$, and $0.05 \%$ bovine serum albumin (BSA). Approximately 40 oocytes were placed between two electrodes and activated with a single direct current (DC) pulse of $1.8 \mathrm{kV} / \mathrm{cm}$ for $30 \mu$ s using an Electro-Cell Fusion apparatus (NEPA gene, Chiba, Japan). Then, the oocytes were incubated in porcine zygote medium-3 (PZM3) containing $1.9 \mathrm{mM} \mathrm{N}$-6-dimethylaminopurine (6-DMAP) for $3 \mathrm{~h}$. Then 15 to 20 embryos were cultured in $40 \mu \mathrm{L}$ of PZM-3 droplets overlaid with light mineral oil (FUJIFILM Irvine Scientific, Santa Ana, USA) for 7 days at $38.5^{\circ} \mathrm{C}$ with $5 \% \mathrm{CO}_{2}$. The cleavage and development of the embryos were evaluated at 2 and 7 days, respectively.

\section{Experiment design}

In experiment 1 , the effects of cumulus-oocyte denudation and the toxicity of hyaluronidase (Product No. H3506, Sigma-Aldrich), ALT-BC4 (80 U/mL, Alteogen), and Hirax (1,500 IU/ $\mathrm{mL}$, BMI Korea, Uiwang, Korea) for the oocyte were compared. Hyaluronidase from bovine testis was diluted in North Carolina University Medium-23 (NCSU-23) washing medium to a final concentration of $0.1 \%(800 \mathrm{U} / \mathrm{mL})$. ALT-BC4, human recombinant hyaluronidase, and Hirax, ovine testis-derived hyaluronidase, were diluted to a final concentration of $800 \mathrm{U} / \mathrm{mL}$ in NCSU23 washing medium. To denudate, 40-60 COCs were treated with hyaluronidase, ALT-BC4, and Hirax for 10 or $20 \mathrm{~min}$. An $800 \mathrm{U} / \mathrm{mL}$ hyaluronidase treatment for $2 \mathrm{~min}$ was used as a positive control.

Experiment 2 aimed to determine the effects of cumulus-oocyte denudation and toxicity for the oocyte when treated with hyaluronidase, ALT-BC4, and ICSI Cumulase ${ }^{\circledR}$ (Product No. 1612, Origio, Ballerup, Denmark). ALT-BC4 and ICSI Cumulase ${ }^{\circledR}$, recombinant human hyaluronidase, were diluted in NCSU-23 washing medium to a final concentration of $80 \mathrm{U} / \mathrm{mL}$. Then, $40-60$ COCs were denuded by pipetting with $800 \mathrm{U} / \mathrm{mL}$ hyaluronidase, $80 \mathrm{U} / \mathrm{mL}$ ALT-BC4, and ICSI Cumulase $^{\circledR}$ for 2 or 20 min. COCs denudated in the NCSU-23 washing medium were used as a negative control and COCs from the $800 \mathrm{U} / \mathrm{mL}$ hyaluronidase treatment for 2 min were used as a positive control.

After denuding, the effects of denudation were confirmed by the degree to which the cumulus cells were attached to the oocyte. The matured oocytes were subjected to parthenogenesis using electrical activation. The toxicity of the various denuding treatments was confirmed by monitoring the developmental ability of the embryo over 7 days. 


\section{Statistical analysis}

For each experiment there were at least 3 replicates, and the results reflect the mean \pm SEM. Data were analyzed using one-way analysis of variance (ANOVA) and Fisher's protected least significant difference (LSD) using SPSS statistics 24 software (IBM SPPS statistics, Chicago, IL, USA). A $p$-value $<0.05$ was considered statistically significant.

\section{RESULTS}

\section{Comparisons of hyaluronidase, ALT-BC4, and Hirax}

In experiment $1, \mathrm{COCs}$ were exposed to $800 \mathrm{U} / \mathrm{mL}$ hyaluronidase derived from bovine-testis (S), Hirax derived from ovine-testis $(\mathrm{H})$, and ALT-BC4 of recombinant human hyaluronidase (A) for 10 or $20 \mathrm{~min}$. The cumulus cells were removed while in each of the three chemicals using pipetting. Fig. 1 shows the denudation of the cumulus cells from the oocytes after exposure to the three hyaluronidases. All groups showed similar denuding levels. There was no degeneration of the oocytes with the different exposure times to the hyaluronidase, ALT-BC4, or Hirax. After denuding, the oocytes were induced to parthenogenesis, and embryonic development was observed
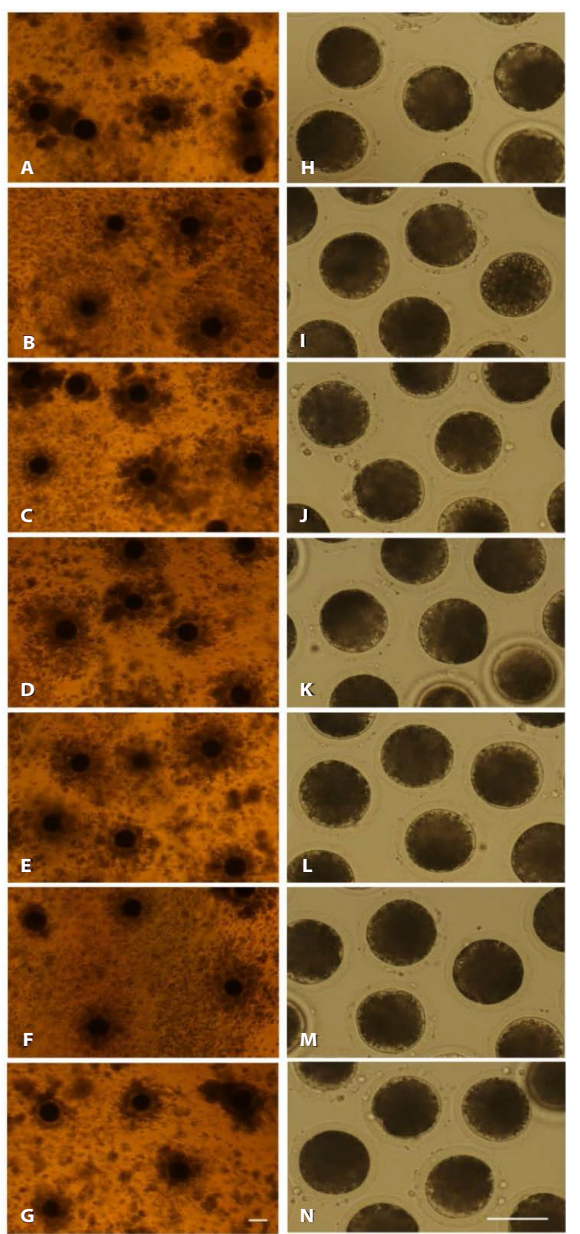

Fig. 1. The denudation of cumulus cells from oocyte using $800 \mathrm{U} / \mathrm{mL}$ hyaluronidases, ALT-BC4 and Hirax. (A-G) Cumulus oocyte complex. $(\mathrm{H}-\mathrm{N})$ denuded oocytes. $(A, H) 2 \mathrm{~S}$, Sigma hyaluronidase treatment for $2 \mathrm{~min}$ (positive control). (B, I) 10S, Sigma hyaluronidase treatment for $10 \mathrm{~min}$. (C, J) 20S, Sigma hyaluronidase treatment for 20 min. (D, K) 10A, ALT-BC4 treatment for 10 min. (E, L) 20A, ALT-BC4 treatment for 20 min. (F, M) $10 \mathrm{H}$, Hirax treatment for $10 \mathrm{~min}$. (G, N) 20H, Hirax treatment for $20 \mathrm{~min}$. Bar $=100 \mu \mathrm{m}$. 
for 7 days (Table 1). There were no significant differences between the developmental abilities of the groups. The blastocyst rate of the hyaluronidase group decreased with the longer treatment time, but those of the ALT-BC4 and Hirax groups were similar regardless of the treatment time.

\section{Comparisons of hyaluronidase, ALT-BC4, and ICSI Cumulase ${ }^{\circledR}$}

In experiment 2, cumulus cells were denuded from oocytes using $800 \mathrm{U} / \mathrm{mL}$ hyaluronidase, and 80 U/mL ALT-BC4 and ICSI Cumulase ${ }^{\circledR}$ for 2 or 20 min. As shown in Fig. 2, almost all cumulus

Table 1. Toxicity of hyaluronidase, ALT-BC4, and Hirax in relation to embryonic development

\begin{tabular}{|c|c|c|c|c|c|c|c|}
\hline \multirow{2}{*}{ Group ${ }^{1)}$} & \multirow{2}{*}{ No. of oocytes } & \multicolumn{6}{|c|}{ Rate of embryo development (\%) } \\
\hline & & 2-cell & 4-cell & 8-cell & 16-cell & Morula & Blastocyst \\
\hline $2 S^{2)}$ & 107 & $96.0 \pm 2.0$ & $84.0 \pm 4.7$ & $70.4 \pm 8.0$ & $58.9 \pm 3.5$ & $52.6 \pm 4.9$ & $52.6 \pm 4.5$ \\
\hline $20 S$ & 104 & $96.9 \pm 2.0$ & $85.2 \pm 4.1$ & $65.5 \pm 8.3$ & $51.8 \pm 9.8$ & $47.7 \pm 9.5$ & $47.7 \pm 9.5$ \\
\hline $10 \mathrm{~A}$ & 115 & $95.8 \pm 2.3$ & $85.9 \pm 5.6$ & $70.3 \pm 9.9$ & $59.2 \pm 10.4$ & $54.0 \pm 9.5$ & $53.3 \pm 8.7$ \\
\hline $20 \mathrm{H}$ & 116 & $94.9 \pm 0.7$ & $89.2 \pm 1.9$ & $77.8 \pm 4.6$ & $57.5 \pm 4.8$ & $50.4 \pm 8.2$ & $50.4 \pm 8.2$ \\
\hline
\end{tabular}

${ }^{11}$ The cumulus-oocyte complexes (the COCs) were denuded using $800 \mathrm{U} / \mathrm{mL}$ hyaluronidase for 10 (10S) and $20 \mathrm{~min}$ (20S); ALT-BC4 for 10 (10A) and $20 \mathrm{~min}$ (20A); and Hirax for 10 $(10 \mathrm{H})$ and $20 \mathrm{~min}(2 \mathrm{OH})$. Values are expressed as the mean $\pm \operatorname{SEM}(n=3)$.

${ }^{2)}$ Positive control (2S), the traditional denuding method that removed cumulus cells from oocytes using $800 \mathrm{U} / \mathrm{mL}$ hyaluronidase for $2 \mathrm{~min}$.

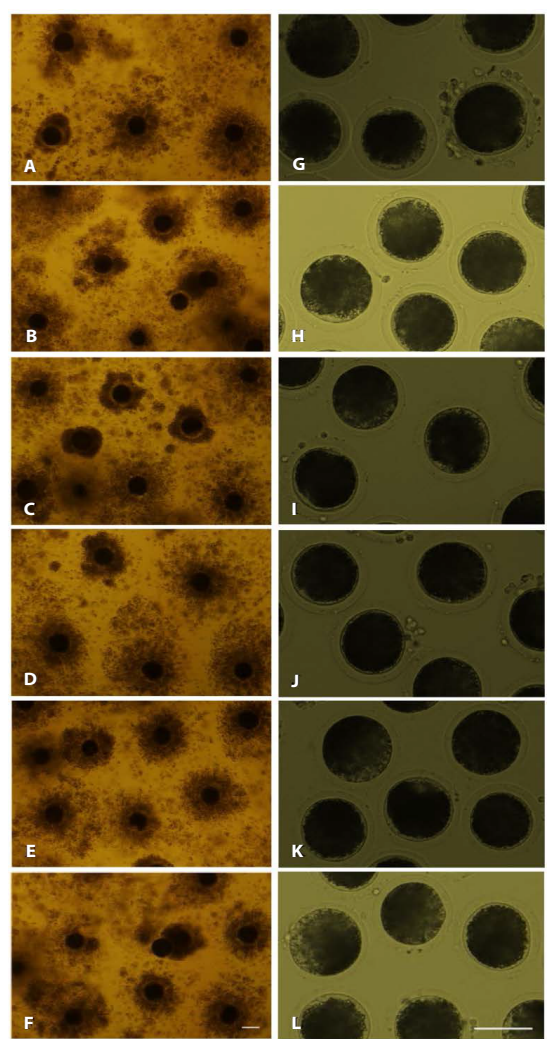

Fig. 2. Effect of hyaluronidase, ALT-BC4 and ICSI cumulase ${ }^{\circledR}$ on the denudation. (A-F) Cumulus oocyte complex. (G-L) denuded oocytes. (A, G) V, COCs were denuded in the NCSU-23 washing medium by pipetting (negative control). (B, H) 2S, $800 \mathrm{U} / \mathrm{mL}$ Sigma hyaluronidase treatment for $2 \mathrm{~min}$ (positive control). (C, I) $2 \mathrm{~A}, 80$ U/mL ALT-BC4 treatment for $2 \mathrm{~min}$. (D, J) 20A, $80 \mathrm{U} / \mathrm{mL}$ ALT-BC4 treatment for $20 \mathrm{~min}$. (E, K) 2C, $80 \mathrm{U} / \mathrm{mL}$ ICSI cumulase ${ }^{\circledR}$ treatment for $2 \mathrm{~min}$. $(\mathrm{F}, \mathrm{L}) 20 \mathrm{C}, 80 \mathrm{U} / \mathrm{mL}$ ICSI cumulase ${ }^{\circledR}$ treatment for $20 \mathrm{~min}$. Bar $=100 \mu \mathrm{m}$. 
cells were removed from the oocytes and they were not degenerated in any of the groups. After denuded oocytes were activated by electrical parthenogenesis, toxicity was investigated based on the developmental ability of the embryos (Table 2, Fig. 3). In embryos produced from the oocytes

Table 2. Toxic effects of hyaluronidase, ALT-BC4, and ICSI Cumulase ${ }^{\circledR}$ on embryonic development

\begin{tabular}{lccccccc}
\hline \multirow{2}{*}{ Group $^{1)}$} & \multirow{2}{*}{ No. of oocytes } & \multicolumn{5}{c}{ Rate of embryo development (\%) } \\
\cline { 3 - 8 } & & 2-cell & 4-cell & 8-cell & 16-cell & Morula & Blastocyst \\
\hline $\mathrm{V}^{2)}$ & 152 & $97.8 \pm 1.2$ & $88.1 \pm 1.7$ & $65.2 \pm 3.6$ & $51.4 \pm 2.0$ & $45.6 \pm 4.0$ & $44.7 \pm 4.7$ \\
$2 \mathrm{~S}^{3)}$ & 158 & $92.6 \pm 0.4$ & $86.1 \pm 3.7$ & $66.2 \pm 2.0$ & $48.3 \pm 4.4$ & $41.6 \pm 4.3$ & $40.7 \pm 3.5$ \\
$2 \mathrm{~A}$ & 155 & $99.2 \pm 0.8^{*}$ & $96.1 \pm 2.1^{*}$ & $77.5 \pm 4.0$ & $63.4 \pm 3.8$ & $63.4 \pm 3.8^{*}$ & $63.4 \pm 3.8^{*}$ \\
$2 \mathrm{~A}$ & 158 & $94.1 \pm 2.4$ & $86.4 \pm 2.8$ & $67.5 \pm 5.0$ & $44.7 \pm 7.2$ & $40.7 \pm 6.7$ & $39.8 \pm 5.8$ \\
$2 \mathrm{C}$ & 152 & $93.6 \pm 3.7$ & $87.0 \pm 3.6$ & $72.7 \pm 9.5$ & $56.4 \pm 9.2$ & $46.5 \pm 7.1$ & $46.5 \pm 7.1$ \\
$20 \mathrm{C}$ & 157 & $96.6 \pm 1.7$ & $94.1 \pm 1.8$ & $84.5 \pm 2.3^{*}$ & $52.0 \pm 2.3$ & $47.2 \pm 3.7$ & $43.7 \pm 2.7$ \\
\hline
\end{tabular}

${ }^{11}$ The COCs were exposed to $800 \mathrm{U} / \mathrm{mL}$ hyaluronidase for $2 \mathrm{~min}(2 \mathrm{~S}) ; 80 \mathrm{U} / \mathrm{mL}$ ALT-BC4 for 2 (2A) and $20 \mathrm{~min}(20 \mathrm{~A})$; and $80 \mathrm{U} / \mathrm{mL}$ ICSI Cumulase® for 2 (2C) and 20 min (20C). Results are expressed as the mean \pm SEM $(n=3)$.

${ }^{2)}$ Negative control (V), the cumulus-oocyte complexes (the COCs) were pipetted in the NCSU-23 washing medium for denuding.

${ }^{33}$ Positive control (2S), the traditional denuding method that removed cumulus cells from the oocytes using $800 \mathrm{U} / \mathrm{mL}$ hyaluronidase for $2 \mathrm{~min}$.

*Values differ significantly compared with the positive control $(2 S)(p<0.05)$.
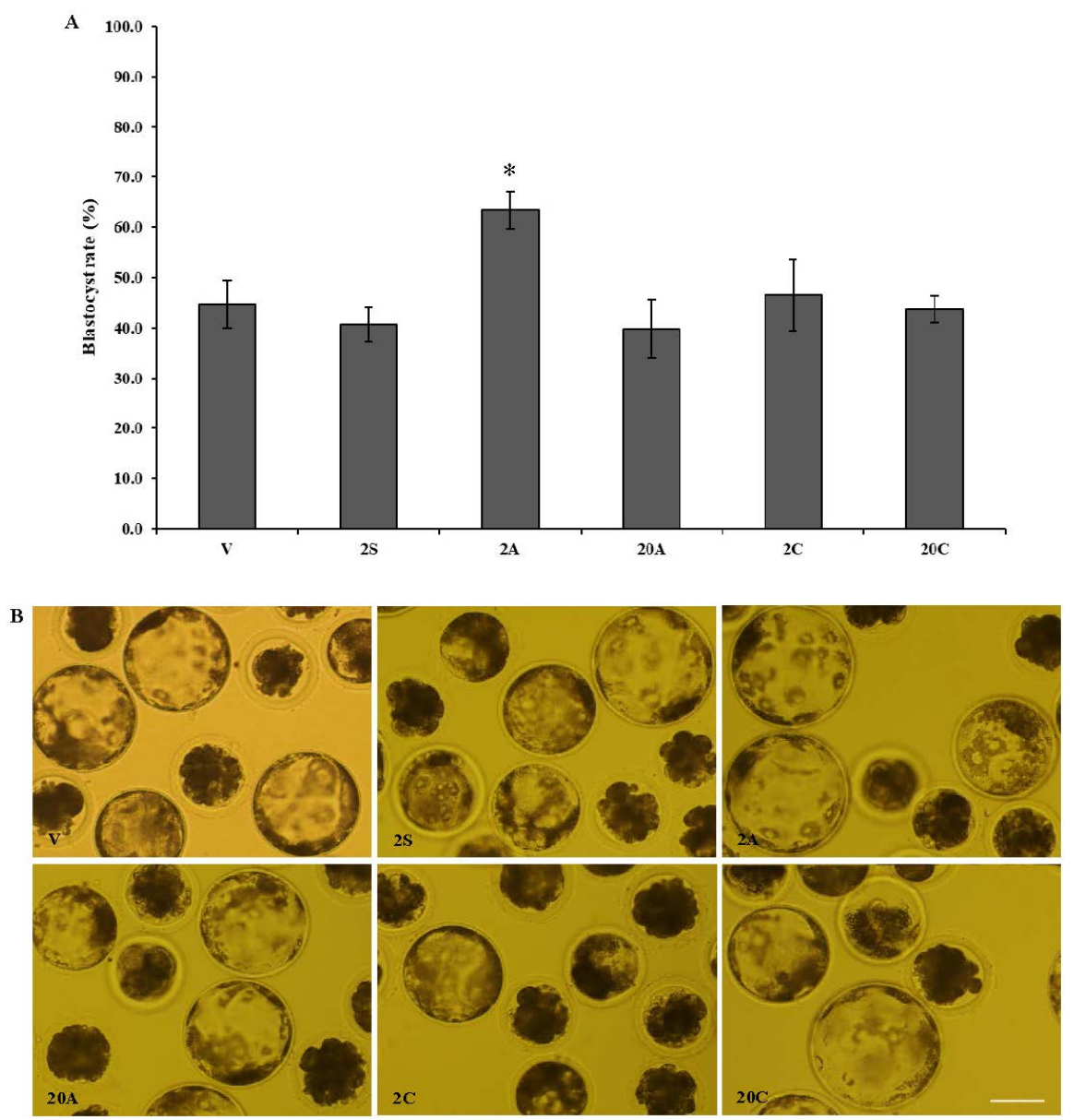

Fig. 3. Effect of hyaluronidase, ALT-BC4 and ICSI cumulase ${ }^{\circledR}$ on the embryonic development. (A) Blastocyst rate of parthenogenetic embryos using denuded oocyte by hyaluronidase, ALT-BC4 and ICSI cumulase ${ }^{\circledR}$. (B) Images of cultured embryos for 7 days. ${ }^{*}$ Values differ significantly compared with the positive control $(2 S)(p<0.05)$. Bar $=100 \mu \mathrm{m}$. 
denuded by ALT-BC4 for 2 min (2A), the rates of the 2, 4, morula, and blastocyst stages were significantly higher than in the positive control (2S). However, embryonic development in the other groups was similar. After the 20 min treatments, there were no significant differences in the blastocyst rates among the ALT-BC4, ICSI Cumulase ${ }^{\circledR}$, and control.

\section{DISCUSSION}

Oocyte denudation is a necessary part of in vitro embryo production. It cannot be determined after IVM if oocytes are mature or immature, as they are surrounded by cumulus cells in the COCs. In this study, the removal of cumulus cells and cytotoxicity were examined in porcine COCs treated with ALT-BC4, a novel human recombinant hyaluronidase, and the results were compared to those using animal-derived hyaluronidases (bovine and ovine) and another human recombinant hyaluronidase (ICSI Cumulase ${ }^{\circledR}$ ). The cumulus cells were denuded using the hyaluronidases by pipetting for a short time. The denuded oocytes were confirmed by the extrusion of the first polar body, which indicates maturation [12]. Previous studies have reported the effect of hyaluronidase on the quality of human or mice oocytes during cumulus cell removal. The present study compared the effect of different sources of hyaluronidases on porcine oocytes.

In experiment 1 , we used bovine-derived, ovine-derived (Hirax), and human recombinant (ALT-BC4) hyaluronidases for 10 and $20 \mathrm{~min}$. Generally, for the ICSI procedure, the hyaluronidase treatments to remove cumulus cells are less than 1 minute, but we exposed COCs to hyaluronidase for up to $20 \mathrm{~min}$ to confirm its toxicity. In this study, we used $800 \mathrm{U} / \mathrm{mL}(0.1 \%$, w:v) hyaluronidase as this concentration has been adapted for porcine COC removal [13]. From our preliminary experiment, it was confirmed that the rate of oocyte degeneration was similar for oocyte denudation with all three hyaluronidases (data not shown). Hirax and ALT-BC4 were first used to denude oocytes in pigs. After denudation, oocytes of all groups showed similar degeneration and denuding levels. In addition, there were no significant differences in the development of the parthenogenetically activated embryos amongst the different groups. In the Sigma hyaluronidase groups, as the exposure time increased, the rate of embryo development decreased. However, in the ALT-BC4 and Hirax groups, the developmental ability of oocytes was similar. In conclusion, it was confirmed that when oocyte cells were exposed to ALT-BC4 for a long time ( $20 \mathrm{~min})$, they exhibited cytotoxicity similar to that induced by Sigma hyaluronidase, an animal-derived hyaluronidase, and could thus function as its replacement.

In experiment 2, the effects of the bovine-derived and recombinant human (ALT-BC4 and ICSI Cumulase ${ }^{\circledR}$ ) hyaluronidases for 2 and 20 min were compared in terms of oocyte denudation and quality. ICSI Cumulase ${ }^{\circledR}$ is the only human recombinant hyaluronidase currently approved by the U.S. Food and Drug Administration (FDA) and European Medicines Agency (EMA) for ICSI procedures. From the results of exp. 1, the embryonic development of the hyaluronidase treatment for 2 and 10 min was found to be similar, and this resulted in the treatment times of 2 and 20 min being selected for this investigation. Hyaluronidase $(80 \mathrm{U} / \mathrm{mL})$ is widely used in clinical settings to denude human oocytes [12]; therefore, porcine COCs were treated with $80 \mathrm{U} / \mathrm{mL}$ ALT-BC4 and ICSI Cumulase ${ }^{\circledR}$. As with the results of exp. 1, oocytes of all groups were completely denuded, and the rate of degeneration was similar. However, a high blastocyst rate was obtained when oocytes were exposed to ALT-BC4 for 2 min compared with the positive control that was treated with hyaluronidase for $2 \mathrm{~min}$. The other groups had no significant differences.

This study demonstrated the toxicity of diverse sources and exposure times to hyaluronidase for oocytes during denudation. Previously the exposure time and concentration of bovine hyaluronidase were reported to affect oocyte quality [14]. However, human recombinant hyaluronidase 
(Cumulase ${ }^{\circledR}$ ) was found to improve the ICSI survival and fertilization rates [5]. De Vos et al. reported that human recombinant hyaluronidase is not inferior to bovine hyaluronidase in terms of denudation, survivability, fertilization, or the development rate of human oocytes [15]. Likewise, this study indicated that Hirax, ALT-BC4, and ICSI Cumulase ${ }^{\circledR}$ were not harmful to porcine oocytes compared to traditional bovine-derived hyaluronidase. Prolonged exposure to hyaluronidase slightly decreased the developmental ability of the embryo, but there was no significant difference. In particular, the administration of $80 \mathrm{U} / \mathrm{mL}$ ALT-BC4 for 2 min improved embryonic development. In conclusion, ALT-BC4, a human recombinant hyaluronidase, was found to be effective for oocyte denudation prior to microinjection, ICSI, and SCNT. Although animal-derived hyaluronidases are used to reduce the cost, they carry several risks from impurities as they are extracted from the testicles of animals and may contain various other proteins. Human recombinant hyaluronidase has a higher purity as it is expressed in $\mathrm{CHO}$ cells and purified. Especially, ALT-BC4 has improved productivity, which results in cost efficiency for production. The process of removing cumulus cells from oocytes is an essential process in the ICSI procedure and thermal stability, and the purity of ALT-BC4 is better than that of animal-derived hyaluronidase. Thus, these results suggest that ALT-BC4 could be advantageous for use in ICSI procedures for human fertilization.

\section{REFERENCES}

1. Stern R. Devising a pathway for hyaluronan catabolism: are we there yet? Glycobiology. 2003;13:105r-15r. https://doi.org/10.1093/glycob/cwg112

2. Camaioni A, Salustri A, Yanagishita M, Hascall VC. Proteoglycans and proteins in the extracellular matrix of mouse cumulus cell-oocyte complexes. Arch Biochem Biophys. 1996;325:190-8. https://doi.org/10.1006/abbi.1996.0024

3. Abdul-Aziz M, MacLusky NJ, Bhavnani BR, Casper RF. Hyaluronidase activity in human semen: correlation with fertilization in vitro. Fertil steril. 1995;64:1147-53. https://doi. org/10.1016/S0015-0282(16)57976-X

4. Taylor TH, Elliott T, Colturato LF, Straub RJ, Mitchell-Leef D, Nagy ZP. Comparison of bovine-and recombinant human-derived hyaluronidase with regard to fertilization rates and embryo morphology in a sibling oocyte model: a prospective, blinded, randomized study. Fertil steril. 2006;85:1544-6. https://doi.org/10.1016/j.fertnstert.2005.10.053

5. Evison M, Pretty C, Taylor E, Franklin C. Human recombinant hyaluronidase (Cumulase ${ }^{\circledR}$ ) improves intracytoplasmic sperm injection survival and fertilization rates. Reprod biomed online. 2009;18:811-4. https://doi.org/10.1016/S1472-6483(10)60030-2

6. Bookbinder L, Hofer A, Haller M, Zepeda M, Keller GA, Lim J, et al. A recombinant human enzyme for enhanced interstitial transport of therapeutics. J Control Release. 2006;114:230-41. https://doi.org/10.1016/j.jconrel.2006.05.027

7. Martin-Deleon PA. Germ-cell hyaluronidases: their roles in sperm function. Int J Androl. 2011;34:e306-18. https://doi.org/10.1111/j.1365-2605.2010.01138.x

8. Itagaki Y, Toyoda Y. Effects of prolonged sperm preincubation and elevated calcium concentration on fertilization of cumulus-free mouse eggs in vitro. J Reprod Dev. 1992;38:219-24. https://doi.org/10.1262/jrd.38.219

9. Ishizuka Y, Takeo T, Nakao S, Yoshimoto H, Hirose Y, Sakai Y, et al. Prolonged exposure to hyaluronidase decreases the fertilization and development rates of fresh and cryopreserved mouse oocytes. J Reprod Dev. 2014;60:454-9.

10. Furuhashi K, Goto S, Hashimoto H, Matsumoto Y, Kokeguchi S, Shiotani M. Comparison of bovine- and recombinant human-derived hyaluronidase in ICSI patients. J Mamm Ova Res. 
2010;27:61-4. https://doi.org/10.1274/jmor.27.61

11. Lin T, Diao YF, Choi HS, Oqani RK, Kang JW, Lee JE, et al. Procedure used for denuding pig oocytes influences oocyte damage, and development of in vitro and nuclear transfer embryos. Anim Reprod Sci. 2015;152:65-76. https://doi.org/10.1016/j.anireprosci.2014.11.009

12. de Moura BRL, Gurgel MCA, Machado SPP, Marques PA, Rolim JR, de Lima MC, et al. Low concentration of hyaluronidase for oocyte denudation can improve fertilization rates and embryo quality. JBRA Assist Reprod. 2017;21:27-30. https://doi.org/10.5935\% 2F1518-0557.20170008

13. Kim KJ, Chun JL, Lee KB, Lee JH, Park KS, Han KW, et al. Effect of acteoside on the re-localization and abnormal morphology of mitochondria in porcine oocytes during in vitro maturation.J Assist Reprod Genet. 2016;33:939-48. https://doi.org/10.1007/s10815-016-0729-x

14. Van de Velde H, Nagy ZP, Joris H, De Vos A, Van Steirteghem AC. Effects of different hyaluronidase concentrations and mechanical procedures for cumulus cell removal on the outcome of intracytoplasmic sperm injection. Hum Reprod. 1997;12:2246-50. https://doi.org/10.1093/ humrep/12.10.2246

15. De Vos A, Van Landuyt L, Van Ranst H, Vandermonde A, D'Haese V, Sterckx J, et al. Randomized sibling-oocyte study using recombinant human hyaluronidase versus bovine-derived Sigma hyaluronidase in ICSI patients. Hum Reprod. 2008;23:1815-9. https://doi.org/10.1093/ humrep/den212 